\title{
Una ricerca del contagio vivo agli albori dell'Ottocento
}

\author{
Di Luigi Belloni, Milano
}

Nella prima metà dell'Ottocento, nel Paese dov'eran morti da poco Morgagni e Spallanzani, la medicina fu dominata da una ripresa della concezione metafisica, sistematica e dogmatica che per circa mezzo secolo paralizzò quasi completamente la ricerca obiettiva ${ }^{1}$. La sterilità dell'osservazione e dell'esperimento s'accompagnò allora con la prolissità degli scritti e con l'acrimonia d'infinite polemiche e di feroci attacchi personali, tra il cozzare violento di dottrine prive o povere di vero contenuto scientifico.

Tipico rappresentante di quel periodo nero della medicina italiana fu GIOVANNI RASORI (1766-1837) ${ }^{2}$ : scrittore brillante, polemista vivace, uomo d'azione ricco d'intelligenza e d'ambizione, dotato di capacità letterarie e di talento poetico. La sua teoria del controstimolo, che per semplicità e logicità sedusse molti tra $i$ medici più sperimentati, esercitò tale influenza da scindere i medici dell'Alta Italia in due opposti partiti: rasoriani e antirasoriani.

Nella sua ultima opera ${ }^{3}$, il RASORI riassume con queste parole le proprie concezioni sulla natura dei contagi: «posseggono tre proprietà costanti ed essenziali, ciò sono: di propagarsi diremmo all'infinito, dove trovino pascolo al loro sviluppo; di conservare mai sempre nel propagarsi l'identità della specie; di riprodursi pei loro semi quando siano applicati là dove non mai esistettero, o da gran pezza avevano cessato di esistere. Ma queste proprietà tutte e tre appartengono alla vita esclusivamente; donde si cava per necessaria conseguenza che i contagi sono materia dotata di vita. Questa dottrina fu da noi esposta fin dal 1796 dalla cattedra di Patologia nella Università di Pavia, e successivamente nei primi anni del secolo presente nella Clinica Militare di Milano.»

La veridicità di questa asserzione è documentata da un membro stesso del partito antirasoriano: Jean-Antoine Ozanam (1773-1837), il francese

${ }^{1}$ A. Cazzaniga, La grande crisi della Medicina italiana nel primo Ottocento, Milano 1951, di p. 122. Questo importante studio critico fu anche pubblicato a puntate nei volumi 4, 5,6 (1948-1950) della rivista "Castalia».

2 A. Monti, Giovanni Rasori nella storia della scienza e dell'idea nazionale. Corsi autunnali per Italiani e Stranieri tenuti nella R. Università di Pavia: lezioni e conferenze dell'anno 1928. Pavia 1929, p. 5-143.

${ }^{3}$ Teoria della flogosi, Milano 1837, vol. I, p. 232. 
laureato a Pavia nel 1810 e autore d'uno scritto ${ }^{4}$, che si presenta come un compendio obiettivo della dottrina rasoriana desunto dalla viva voce del Maestro, ma che in realtà ne costituiva un insidioso attacco ispirato da uno dei maggiori nemici del Rasori: Pietro Moscati (1739-1824). Nel capitolo dell'opera dedicato all'Analogia tra $i$ caratteri dei contagj e degli animali possiamo leggere: «... diremo consistere l'essenza dei contagj in esseri organici d'una propria e determinata specie, i quali si propagano come i pidocchj. Abbiamo un argomento di più nel contagio della rogna che si sa non dipendere da altro che da un insetto. La teoria della generazione spontanea degli insetti, degli animali infusorj è falsa. Viene provato che non nascono insetti ove non fu prima deposizione di uova. Lo stesso dicasi dei contagj; e quantunque il microscopio non abbia fatto ancora conoscere le uova, o germi, o animali costituenti i rispettivi contagj, non perciò si deve rinunciare ad una ipotesi fondata su i fatti. Ne abbiamo però abbastanza per istabilire quel corollario, che l'essenza dei contagj consiste in particolari corpi organici propagantisi fra gli animali.» E più avanti leggiamo che è proprietà dei contagi di potersi «conservare mesi ed anni in istato inattivo, e svilupparsi al momento che trovansi nelle opportune circostanze, come il Rotifero, sentendo l'umido».

Tralascio la critica a questi concetti fatta dall'Ozanam, per ricordare come la dottrina del contagio vivo procurasse al RASORI taccia di visionario persino presso i suoi più fedeli seguaci. Tale fu Giuseppe Del Chiappa (1782-1866), che del Rasori con devozione d'allievo stese la biografia ${ }^{5}$ e trascrisse l'epistolario ${ }^{6}:$ «Nè voglio far mostrare le false ed erronee dottrine che egli professava intorno alle febbri intermittenti ed al miasma e della cura quindi che credeva dovesse unicamente convenir loro: ma questo fu il debole di quel grand'uomo.»

\footnotetext{
${ }^{4}$ I.A.F. Ozanam, Cenni sulla teoria e la pratica della dottrina medica del controstimolo, con alcune osservazioni comparative tra $i$ risultamenti del metodo di cura secondo $i$ principi di questa dottrina, e quelli del metodo comunemente adottato, Milano 1812 (1 ed.), p. 33-6. L'opera ebbe una seconda edizione (Milano 1816). L'Ozanam è anche autore della Histoire médicale générale et particulière des maladies épidémiques, contagieuses et épizootiques, qui ont régné en Europe depuis les temps les plus reculés, et notamment depuis le $X I V^{e}$ siècle jusqu'à nos jours, Paris-Lyon, 1817-1823, 5 vol. Nel vol. III (1823) a p. 188-95 è descritta l'oftalmia di Ancona.

${ }^{5}$ G. Del Chiappa, Della vita di G. Rasori libri sei, Milano 1838.

${ }^{6}$ Cod. Parmense 936 (Bibliot. Palatina di Parma), in fol. p., di ff. 131, intitolato «Lettere familiari di Giovanni Rasori,illustre medico e letterato, raccolte e copiate dagli autografi da Giuseppe Del Chiappa. In Pavia, nell'anno 1844». Cf. Frati, l. c., nota 1 a p.11.
} 
Ben diverso fu il giudizio di Agostino Bassi (1773-1856), il dottore in legge passato alla storia della biologia par aver dimostrato nel 1835/36 che il «mal del calcino» del baco da seta proveniva dall'infezione dell'insetto da parte d'un altro essere vivente, un Ifomicete che in suo onore fu chiamato «Botrytis Bassiana». ${ }^{7}$ Nel 1846 il BAssi ci racconta d'aver contratto stretta amicizia col RASORI fin dall'epoca dei propri studi universitari a Pavia, «per il che, andando spesso a Milano a ritrovarlo, mi tratteneva per lo più con esso in lunghi discorsi segnatamente dopo la mia scoperta sul Calcino, a cui comunicai molto tempo prima che la rendessi di pubblica ragione. Appena udito che ebbe il caro amico la supposta e da me riconosciuta natura del calcino, mi disse: ,Io sono pienamente persuaso della verità dell'utile vostro trovamento: sono già molti anni ch'io porto opinione che le febbri intermittenti vengono prodotte da parassiti che ne rinnovano l'accesso all'atto della loro riproduzione, la quale succede più o meno presto secondo le diverse loro specie.' Perciò, egli mi diceva, nasce la febbre intermittente, quotidiana, terzana e quartana.» Gustoso il seguente episodio raccontatoci dal BASSI qualche riga più avanti: «Tenendo un giorno un lungo sermone collo stesso RASORI intorno alla mia opinione che tutte le malattie attaccaticcie siano prodotte da esseri viventi parassiti animali o vegetali, terminata che fu la conferenza, la quale durò da circa tre ore, dandomi egli comiato, col prendermi per mano, mi disse: se altri avessero per avventura uditi questi nostri discorsi, eccoli uniti i due matti' avrebbero esclamato». ${ }^{8}$

Fu questa probabilmente la ragione per cui il RASORI, che anche nell'epi-

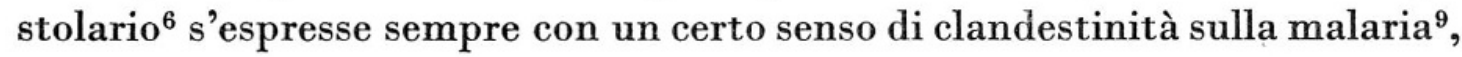
non sviluppò a fondo nelle opere a stampa le proprie concezioni sulla natura vivente dei contagi.

La più interessante documentazione viene quindi ad esser costituita dall'operetta d'un suo ardente seguace, il medico veronese Francesco

${ }^{7}$ L. MüNster, Ein vergessener Vorkämpfer der Parasitenlehre: Agostino Bassi aus Lodi. Zugleich ein Vergleich der Tätigkeit Bassis, Listers und Semmelweis's und ein Rückblick auf die Entwicklung des Gedankens über Contagium Animatum, Janus 37, 221-46 (1933).

${ }^{8}$ A. BASSI, Discorsi sulla natura e cura della pellagra ... e rimedj sicuri e pronti contro le febbri intermittenti ..., Milano 184.6, p. 32/3. I brani sono riprodotti a p. 357/8 di Opere di Agostino Bassi, scelte e pubblicate a cura del Comitato Nazionale per la Ristampa, auspice la Società Medico-Chirurgica di Pavia, Pavia 1925.

๑ F. Marimò, Giovanni Rasori e la malaria, Rendic. Ass. Med.-Chir. Parma 1, 162-6 (1900). Sull'argomento cf. anche U. FAUcci, L'idea di Giovanni Rasori sulla natura della malaria. Riv. Storia Scienze Med. Natur. 30, 177-87 (1939). 
VaSani (1782-1850) ${ }^{10}$, intitolata Storia dell'ottalmia contagiosa dello Spedale militare d'Ancona: origine e natura di quel contagio ed alcuni cenni intorno ai comuni errori nella terapeutica dell'ottalmia cronica (Verona 1816, di p.140). Apre lo scritto una dedica «Al mio Maestro», facilmente identificabile nel RASORI, che non viene esplicitamente nominato, perchè trovavasi allora in carcere per motivi politici. Infatti il RASORI è uno dei primi martiri dell'indipendenza e dell'unità d'Italia. Nella notte dal 3 al 4 dicembre 1814 venne arrestato dagli Austriaci per aver partecipato alla «cospirazione militare» e rimase in carcere, prima a Mantova e poi a Milano, fino al 9 marzo $1818^{11}$. $\mathrm{Al}$ momento dell'arresto egli versava in estrema povertà e durante la lunga prigionia lasciò in gravissime ristrettezze finanziarie la giovane ed unica figlia SABINA. Si tratta d'un particolare biografico che ci riuscirà utile più avanti, in rapporto all'affermazione del RıMA circa la paternità della redazione della Storia del VASANI.

Questo scritto si riferisce a una terribile epidemia oftalmica che nel 1812/13 infierì sulle truppe della guarnigione d'Ancona, riempiendo l'ospedale militare di quella città e accecando molti soldati. L'epidemia cominciò a manifestarsi e rimase dapprima limitata al VI Reggimento di linea, ma dopo qualche tempo si diffuse alle altre truppe della guarnigione. La natura contagiosa non fu riconosciuta nei primi rapporti inviati da quei medici militari al Ministero della Guerra, che risiedeva a Milano, capitale del Regno Italico. I continui progressi dell'epidemia impensierirono non poco il Ministero e soprattutto C. G. ANNibale OModei (1779-1840)12, che ricopriva la carica di «medico consulente». L'OMODEI richiese il parere d'un influente medico, il Rasori, e d'un non meno influente chirurgo, Antonio Scarpa $(1752-1832)^{20}$, entrambi pronunciatisi per la natura contagiosa dell'oftalmia,

10 Il VASANI nacque e morì a Brenzone sul Garda (provincia di Verona) e fu medico municipale di Verona. Queste notizie, al pari delle date di nascita e di morte, furono desunte da documenti dell'Archivio di Stato di Verona per cortese interessamento del prof. FraNcesco Pellegrini.

${ }^{11}$ C. Frati, Ricordi di prigionia, memorie autobiografiche e frammenti poetici di Giovanni Rasori (vol. IX della Biblioteca di Storia italiana recente, 1800-1870, edita dalla R. Deputazione sovra gli studi di Storia Patria per le Antiche Provincie e la Lombardia), Torino 1921, p. 1-132. L'opera contiene anche un «Saggio di bibliografia rasoriana (1794-1913)».

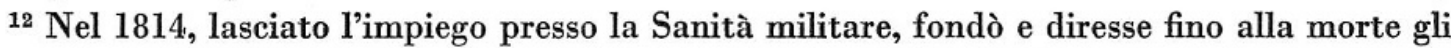
«Annali di Medicina Straniera», trasformatisi nel 1817 negli «Annoli Universali di Medicina». Per la biografia e il ritratto cf. C.A. Calderini, Cenni biografici sul dottore Annibale Omodei, Ann. Univ. Med. 93, I-XXIV (1840). 
e dispose perchè venisse inviato sul luogo un ispettore di sanità nella persona di Tommaso Rima (1775-1843) ${ }^{13}$, che giunse in Ancona il 19 novembre 1812 proveniente dall'ospedale militare di Mantova. Nel gennaio del 1813 arrivò in Ancona quale «medico requisito provvisorio», cioè quale subalterno, anche il VASANI: aggregato al reparto degli ottalmici, vi rimase fino al 20 maggio dello stesso anno. Ed ora seguiamo il suo racconto.

Il VI Reggimento era stato continuamente, benchè in forma attenuata ed accessionale, bersagliato dall'oftalmia fin dal 1805, allorquando i suoi uomini giunsero in contatto, nell'isola d'Elba, con un reparto francese duramente provato dalla malattia nel corso della spedizione d'Egitto. Trattasi quindi della celebre «oftalmia egiziana», triste retaggio della campagna d'Oriente (1798/99), sulla cui storia e natura, substrato batteriologico compreso, non mi diffondo dopo quanto sull'argomento fu scritto con competenza forse unica da Max Meyerhof (1874-1945) ${ }^{14}$.

Dato che la natura dei contagi è tale «che conservandosi sempre gli stessi, si diffondono mostrando una maggiore o minore facilità di propagazione, una maggiore o minore intensità e ferocia nei diversi anni, nelle diverse stagioni, nei diversi corpi su' quali agiscono, e in somma nelle diverse circostanze a noi ancora sconosciute, ma che sono o propizie o contrarie ai semi contagiosi», era logico arguire che questi ultimi avessero trovato in Ancona le «propizie circostanze per propagarsi ed infierire più del solito» (p. 34).

L'epidemia rimase in un primo tempo strettamente limitata al VI Reggimento e risparmiò il IV Reggimento pure dislocato nell'Anconetano: fatto certamente inspiegabile dagli anticontagionisti, che imputavano l'oftalmia all'influenza del clima. Quando però il IV Reggimento venne a condividere in Ancona la caserma del VI Reggimento, esso pure venne colpito, e con

${ }^{13}$ Nato a Mosogno nel Ticino e compiuti gli studi universitari a Roma, percorse la carriera di chirurgo nell'esercito napoleonico italiano. Fu in seguito chirurgo primario a Ravenna e, dal 1822, a Venezia, dove morì. Scoprì il circolo refluo del sangue nelle varici. Cf. D. Giondano, Nel centenario della dottrina di Tommaso Rima su le varici ..., Venezia 1925, di p. 48 e ritratto; ristampato in D. GIORDANo, Scritti e discorsi pertinenti alla storia della medicina ..., Milano 1930, p. 270-95 (L. BELLONI in Ricerche, scoperte e invenzioni di medici svizzeri [Esposizione di libri e manoscritti organizzata con l'appoggio della Ciba Société Anonyme, Basilea, dalla Biblioteca Cantonale di Lugano], Basilea 1947, Append. no 3, p. 5/6). Sul Rima quale idroterapeuta cf. L. BellonI, I bagni galleggianti d'un chirurgo dell'Ottocento, Rivista Ciba 2 (fasc. 10), 343/4 (1948).

${ }^{14}$ A Short History of Ophthalmia during the Egyptian Campaigns of 1798-1807, Brit. J. Ophthalm. 16, 129-52 (1932). 
tremenda violenza, dalla malattia. I dati epidemiologici erano quindi tali da «costringere l'osservatore a riconoscere un principio contagioso, e ad escludere qualunque altra cagione» (p. 39). Essendo poi stato «osservato in Egitto, che questa ottalmia è pur comune agli animali domestici» (p. 40) $)^{15}$, veniva logico il tentativo di riprodurre artificialmente la malattia inoculando il pus dell'uomo malato nell'animale.

«Presi adunque due cagnoletti, e mediante alcuni pannolini intrisi dell'umore ottalmico infettai loro gli orli delle palpebre. Quattro giorni dopo ebbero gli occhi gonfi e cisposi, con tutti i sintomi della dominante affezione ottalmica. Io mi disfeci d'amendue questi animali, quando vidi che la malattia continuava, con tutto che gli avessi lavati molte e molte fiate in un recipiente d'acqua.

In quest'acqua medesima mi venne in animo d'immergere un terzo cane, nel quale pure osservai svilupparsi una vera idrottalmitide. Ognuno converrà, che questi tre casi poteano bastare a farmi certo di un'azione contagiosa, ma mi piacque inoltre di sperimentare, se quella materia conservasse la sua attività contagiosa, messa che fosse a contatto in altri modi.

Raccolsi per tanto dai diversi pannolini, de' quali mi era servito coi tre cani summentovati, un pocolino di purulente materia ottalmica già diseccata. Ridottala in polvere, ne cacciai una picciolissima quantità verso l'angolo interno dell'occhio d'un quarto cane, ed anche in questo dopo qualche giorno fecesi vedere l'occhio lagrimoso, e tramandante materia eguale all'altra che sgorgava dagli occhi de' nostri ottalmici. Per quindici giorni la malattia di questo cane rimase circoscritta al solo occhio, nel quale aveva introdotta la materia morbifica, ma poi cominciò a risentirsene anche l'altro; e questo esperimento ho io più volte ripetuto, e sempre o più presto o più tardi ne ottenni il medesimo risultato.

Così pure stropicciando dei pannolini infetti di materia ottalmica già secca, in modo che la polvere cadesse sopra gli occhi di un qualche cane, io vidi nascere lo stesso effetto. Persino ho potuto generare l'ottalmia in un cagnoletto coll'immergerlo nell'acqua dov'erano stati lavati i pannolini adoperati da' nostri ottalmici per nettarsi gli occhi ...

15 Il Vasani cita il lavoro di Antonio Savaresi, Descrizione dell'oftulmia d'Egitto, Cairo VIII (1800), nella traduzione francese inserita a p. 90-9 di R. Desgenettes Histoire médicale de l'Armée d'Orient, Paris, An X, 1802. Riporto integralmente il relativo brano del SAVArEsI: «Les animaux ne sont pas plus exempts que les hommes des maladies des yeux; la plupart des chiens sont aveugles ou borgnes, et beaucoup d'ânes, de chevaux, de bœufs, et de chameaux, ont les yeux tachés ou légèrement affectés» (I. partie, p. 92). 
Ora io mi lusingo, che dopo tutto questo nessuno vorrà mettere in dubbio, come nell'umore separato dalla nostra ottalmia vi fosse propriamente un seme contagioso atto a diffondere e a generar l'ottalmia ad ogni opportuna occasione. Imperocchè si è veduto l'umore ottalmico produrre i suoi effetti dentro gli occhi nel suo stato di fluidità naturale, in quello di combinazione coll'acqua, introdotto negli occhi già disseccato, e fattovi anche cadere in polvere a qualche distanza» (p. 40-2).

Trasportando all'uomo gli esperimenti compiuti sull'animale, il VASANI giunse a identificare «quali siano stati i modi precisi, quali i conduttori pel di cui mezzo il contagio ha potuto propagarsi» e precisamente: « $\overline{\mathrm{E}}$ grande in Ancona la scarsezza dell'acqua, e le caserme non erano provvedute d'altra acqua che delle vasche fatte a contenere quella, che è necessaria alla pulitezza dei soldati. A queste vasche appunto venivano gli ottalmizzati frequentemente a lavarsi gli occhi. Provavano ancora un momentaneo sollievo stropicciandoli, e in mancanza di pannolini ricorrevano alle lenzuola, alle coperte, e a tutto ciò in somma che loro veniva alle mani. Dal che ben si comprende, come le vasche ed i letti erano divenuti altrettanti conduttori di contagio ...» (p. 43/4).

«Ma», si chiede ora il VASANI, «questo contagio, ch'io potei propagare artificialmente, come si propaga coll'innesto il vajuolo ed il vaccino, in che consiste egli mai ? ... A questo proposito io mi ricordava d'aver udito anni addietro l'illustre RASORI risuscitare un'opinione avanzata bensì altre volte, ma senza prove che almeno la rendessero probabile; quella cioè che $i$ contagi consistono in qualche razza di viventi infinitamente piccioli, analoghi agli animali infusorj di cui sono popolati tanti liquidi . . . Se non che egli si limitava a riguardarla come una conghiettura, aspettando che il microscopio felicemente adoperato riponesse una volta la conghiettura nel novero dei fatti. Queste considerazioni furon elle che mi diedero animo a provarmi all'impresa offertami dalle circostanze. Imperocchè io aveva fra le mani la materia, che dovea pur contenere la sua particolare generazione di viventi, se vero è, come lo è in fatti, che alla mercè di essa io propagassi la malattia, e se regge la conghiettura che propagazione di contagio equivalga a propagazione d'animaluzzi infusorj.

Misi primieramente alla prova una gocciola di materia ottalmica alquanto densa sotto d'un acuto microscopio; ed ecco ciò che mi apparve all'occhio. Tutta la massa della gocciola rassomigliava ad un corpo giallognolo, pellucido, composto inegualmente di porzioni consistenti e di porzioni fluide . . . Aderenti alle parti solide, oppure moventisi nel fluido notai alquanti cor- 
puscoli tutti uniformi, i quali ora procedevano orizzontalmente, ora scendevano al fondo del liquido, ora si rialzavano alla superficie. Ingranditi dal microscopio erano all'incirca della dimensione d'un mezzo grano di miglio; la figura loro appariva conica schiacciata, ed il colore alquanto più cupo di quello giallognolo della massa. Per quanto osservassi pazientemente $i$ loro andamenti, potei bensì accertarmi che la base del cono era quella sempre che procedeva innanzi, ma non potei distinguer mai qual sorte di movimenti o contrazioni accadesse nel loro corpo mentre si trasportavano da luogo a luogo.

Nella speranza di vedere maggior copia di corpicciuoli e meglio osservarli, mi procurai una gocciola di materia purulente ottalmica la più fluida, ma restai deluso nella mia espettativa; imperocchè, invece di trovarne un maggior numero, ne trovai due soli, ne'quali riconobbi la medesima figura conica, e il medesimo movimento del procedere innanzi colla base; il colore però era più chiaro e pellucido, ed osservandoli bene non si sarebbero male assomigliati ad una squama di pesce.

Le medesime indagini microscopiche ho istituite sulla materia purulente ottalmica dei cani inoculati, e ne ho ottenuti i medesimi risultati; ed in questa pure ho riscontrato, che più copiosi erano i corpuscoli, allorché la materia era più densa, alla quale sembra che vadano volentieri ad attaccarsi.

Siccome aveva osservato, che l'acqua non avea distrutta, ma bensì conservata la forza contagiosa della materia che vi era stata deposta, così mi parve di esaminare quai fenomeni presentasse la materia purulente allungata con acqua. Fatto perciò questo miscuglio, ed agitatolo bene, colla punta di una penna ne presi una gocciolina, e la sottoposi al microscopio. L'esperimento fu fatto più volte; imperocchè talvolta potei discoprire alcuni dei soliti corpicciuoli, e talvolta non ne vidi punto.

Volendo vedere cosa potesse il freddo e il calore su di essi, feci dei miscugli della materia talora con acqua fredda quasi alla congelazione, talora con acqua riscaldata a diversi gradi. In ciascuno di questi casi una volta o l'altra ho osservato i soliti corpicciuoli, e con questa differenza, che nell'acqua agghiacciata $i$ corpicciuoli o erano precipitati al fondo, o si rimanevano attaccati alle parti mucose solide, ma in un caso e nell'altro senza movimento.

Sottoposta al microscopio la materia ottalmica seccata, e ridotta in polvere, non potei ravvisarvi che qualche lucida particella, di cui però non seppi distinguere la figura. Ma, gittata la polvere in alquanta acqua, da lì 
a poco vidi tumefarsi quelle particelle o granellini, come parevano, e a poco a poco rimanendosi in fondo dell'acqua quel po' di materia purulente ammollitasi, nuotare nell'acqua e muoversi come al solito i corpuscoli conici. Così mi offerivano essi lo stesso fenomeno del rotifero, che, diseccato e ridotto ad una semplice pellicina senza moto e senza vita, riacquista l'uno e l'altra sì tosto che si riponga novellamente nell'acqua. Era interessante il determinare quanto tempo potesse la materia diseccata conservarsi suscettibile di represtinare in vita i suoi corpicciuoli; ed io posso dire di non aver affatto perduta l'occasione d'intraprendere questa indagine, avendo cercato di ottalmizzare dei cagnoletti colla materia purulente de'pannolini che avea presso di me da più di un mese. I cagnoletti furono effettivamente ottalmizzati; ond'è ch'io debbo credere, che il lasso di un mese non avea potuto far perdere a quella materia purulente diseccata la facoltà in discorso; e se quei corpicciuoli ponno essere repristinati in vita dopo un mese, non ripugna che possano esserlo dopo un tempo maggiore, e fors'anche indeterminato» (p. 50-5).

Costatata l'assenza di «que' corpicciuoli o animaluzzi viventi ... nella materia delle comuni ottalmie o sporadiche, o croniche»s e l'incapacità di tale materia a "comunicare il male d'occhi a dei cagnoletti», il VASANI racconta gli esperimenti che avrebbe voluto intraprendere per dimostrare che i corpuscoli sono i veri agenti patogeni dell'oftalmia, anziché semplici «animaluzzi fatti a stare in quell'umore, come in tanti altri liquidi sono fatti a stare altri animaluzzi infusorj. La qual ombra di difficoltà avrei potuto dissipare inoculando alquanti cani con materia fluida, riconosciuta prima col microscopio scevra d'animaluzzi, ed alquanti altri con materia densa, alla quale gli animaluzzi si attengono preferibilmente. I primi avrebbero dovuto non contrarre l'ottalmia, la quale doveva essere contratta dai sècondi : con che si veniva a dimostrare, che della materia ottalmica contagiosa rimane la proprietà del contagio a quella sola a cui rimangono aderenti gli animaluzzi. Ma il tempo e le circostanze non mi permisero di eseguire tutto ciò che avrei potuto divisare. D'altronde poi agli scrupoleggiatori non sarebbe stato gran fattol'insistere di nuovo, che con questo non si dimostrava altro, se non la preferenza che hanno gli animaluzzi per la materia più densa, la quale può esser dessa la produttrice del contagio, senza che quelli v'abbiano parte» (p. 56/7).

Non si può negare che gli esperimenti siano stati condotti con una certa logica. Essi diedero al VASANI l'illusione d'aver scoperto in un essere vivente l'agente patogeno dell'oftalmia e di spiegare attraverso le sue pecu- 
liari condizioni biologiche, paragonabili a quelle del Rotifero, alcune costatazioni derivate dallo studio epidemiologico, come i periodi di latenza dell'epidemia e la trasmissione indiretta attraverso veicoli. Interessante anche la descrizione delle recidive a distanza d'anni negli individui clinicamente guariti e la capacità di questi ultimi di trasmettere il contagio, per cui «è naturale di supporre che sieno rimasti latenti dei semi contagiosi» (p. 91).

Passando alla terapia, s'interrompe la logica del VASANI : anzichè trarre le conseguenze dalle sue osservazioni e ricorrere all'impiego di sostanze capaci d'uccidere gli animaluzzi, egli preferì considerare la malattia come una "grave diatesi di stimolo» a carico dell'intero organismo e trattarla quindi coi «controstimoli», sia generali (salasso, sanguisugio, digitale, purganti, tartaro stibiato, nitro, bagno freddo, dieta rigorosa), che locali (colliri di digitale e di tartaro stibiato). Soltanto di sfuggita viene accennata la possibilità d'una vera e propria terapia causale: «Quanto al collirio d'infuso di digitale, mi sembrò che agisse più prontamente, come se avesse per avventura esercitato qualche azione anche sui semi stessi contagiosi» (p. 84).

Questa incongruenza tra i risultati delle ricerche etiogenetiche e i procedimenti terapeutici ${ }^{16}$ fu subito evidenziata nella recensione dell'opera scritta dall'antirasoriano Ciro Pollini (1782-1833) ${ }^{17}$, il quale giustamente

${ }^{16}$ La stessa discrepanza fra dottrina del contagio vivo e terapia di controstimolo fu rilevata nel Rasori da Ozanam, l. c., p. 126.

${ }^{17}$ Giornale di Medicina pratica (del Cavaliere Valeriano Luigi Brera M. D., Padova) 10, 233-273 (1816), dove il recensore tratta ostilmente anche la Storia singolare d'una febbre miliare con alcune considerazioni sopra questa malattia (Verona 1815, di p. 41) scritta dal VASANI per difendere la terapia di controstimolo da lui applicata in un caso mortale di febbre miliare. Le due recensioni, che furono poi ristampate da M. G. Levi Dizionario classico di medicina ..., Venezia 28, 503-14 (1836), 32, 643-51 (1845), portano la sigla «P.S.», corrispondente a «Poluini Syrus» (cf. G. SANDri, Elogio del Dottor Ciro Pollini, Memorie dell'Accad. d'Agricoltura, Commercio, ed Arti di Verona 14, 5-50, 1833). Il PoLLINI sosteneva che la diatesi generata nell'organismo del malato dal contagio proprio della febbre miliare poteva essere ora iperstenica ed ora astenica, caso quest'ultimo in cui doveva sicuramente riuscire controproducente la terapia di controstimolo. Il VASANI attese ben cinque anni a rispondere, finquando cioè succedette al PollinI di curare con esito infausto un caso di «sinoca gastrica-reumatica» che il VASANI, chiamato a consulto poche ore prima della morte del paziente, interpretò invece come una «febbre miliare». L'aspra polemica è documentata dal succedersi dei seguenti opuscoli (il I e il III del Pollini, mentre il II e il IV, scritti dal VASani, sono sforniti in copertina del nome dell'autore, che però manifesta chiaramente la propria identità nel testo): I. Storia d'una sinoca reumatica gastrica, Verona 1821, di p. 13; II. Alcune osservazioni sulla storia d'una sinoca gastrica reumatica, Verona 1821, di p. 23; III. Esame delle osservazioni 
obiettò che, ammessa la reale presenza degli animaluzzi e il loro significato patogeno, anzichè di semplici ospiti della materia purulenta, «la somma delle cose doveva consistere nel distruggere cotali esseri infesti con rimedj locali, come si adopra nella rogna; conciossiachè ammazzati quelli e cesserebbe ben tosto il turbamento universale, e svanirebbe con molta cura anche l'affezione locale». Aggiunge poi il Pollini: «La lettura dell'opera del VASANI ha in me pure risvegliato il capriccio di osservare al microscopio il virus blenorroico, e la marcia delle ulcere sifilitiche, e dei buboni; ma e nell'uno e nell'altra non mi venne veduto animale di sorta.»

La pubblicazione della Storia dell'ottalmia non risvegliò soltanto le ire degli antirasoriani, ma comunque dei medici che, rivestendo l'uniforme militare, s'erano occupati dell'epidemia d'Ancona e vedevano ora il proprio operato messo in cattiva luce dal VASANI, il cui intento polemico trasparla nel modo più lampante dalla stessa citazione dantesca»... e fieti manifesto / L'error de' ciechi che si fanno duci» (Purg. Cant. XVIII) fregiante il frontespizio dell'opera. La risposta non tardò a venire e si concretò nei Cenni sull'ottalmia contagiosa d'Egitto e sulla sua propagazione in Italia ${ }^{18}$ scritti dall'Oморе ${ }^{12}$ in base a documenti ufficiali del passato Ministero della Guerra. Due anni più tardi apparve la replica e precisamente la Risposta di F. Vasani a ciò che lo riguarda nei Cenni del Dr. Omodei sull'ottalmia contagiosa d'Egitto e sulla sua propagazione in Italia (Verona 1818, di p. 47). Sono costretto a sorvolare sulle parti polemiche e sui feroci attacchi d'ambo gli scritti, per trascrivere il brano dell'OмоDEI che maggiormente interessa ai fini del nostro studio : «Un mese prima che il sig. VASANI giungesse in Ancona, il sig. Rima avea ragguagliato il Ministero di due sperimenti fatti colla materia puriforme raccolta dagli ottalmici innestata agli stessi animali. In uno cui venne introdotta sotto le palpebre, egli osservò nel terzo giorno farsi gonfi e cisposi gli occhi; e nell'altro, cui avea injettata la materia nell'uretra, a capo di un mese, vidde nascere una specie di flusso gonorroico» (p. 281).

Il fatto è confermato dal Rima ${ }^{13}$ stesso nelle memorie autobiografiche (Necrologia del Dr.T.Rima) da lui stese in terza persona negli ultimi anni di

del dottore F. Vasani sulla storia d'una sinoca reumatica gastrica scritta dal Dottore C.Pollini, Verona 1821, di p. XXIX; IV. Breve comentario all'esame delle osservazioni sulla storia d'una sinoca-reumatica-gastrica, Vicenza 1822, di p. 91.

${ }^{18}$ Annali di Medicina Straniera 4 (continuazione), 104-44, 222-88, 324-74 (1816). L'opera figura anche in fascicolo a parte (Milano 1816, di p. 159) e, come risulta dal Lexicon di Hirsch, ebbe un'edizione tedesca (Frankfurt a. M. 1820). 
vita. Dal manoscritto autografo, di cui sto curando l'edizione integrale, trascrivo il brano concernente il soggiorno anconetano del RiMA:

«Dominava fieramente nei militari che formavano la guarnigione di Ancona, e dei contorni una ottalmia contaggiosa, vecchio ed infelice retaggio dei soldati reduci dall'Egitto. E vita, ed occhj vi perdevano in gran numero gli infelici che ne venivano attaccati. Giudicava necessario il Ministero che un esperto ed intelligente chirurgo si portasse sul luogo per darvi i necessarj provvedimenti. Sotto la data del $149^{\text {bre }} 1812$ vi veniva spedito in posta il Rima. Riconosciuto a non dubbitare il carattere contaggioso della malattia, furono prontamente attivate tutte le misure sanitarie incominciando dall'isolamento degli infetti. Da questo radicale provvedimento ne derivò il numero sempre più minorato dei nuovi attaccati, ed in due mesi circa non si avevano che a curare le tristi conseguenze della vinta malattia. Fece sorpresa che dopo due anni sortisse col nome del Dr. VASANI di Verona una pretesa Storia dell'ottalmia contaggiosa di Ancona. Si appropriavano gli esperimenti fatti sugli animali, non che quei riflessi che emergevano dagli ufficiali rapporti che al cessato Ministero della Guerra si comunicavano dal Professore Rima nominato allora Ispettore di sanità di quel circondario. Commetteva egli al Dr. VASANI mandatogli qual medico soprannumerario (essendovi già gli attivi BuSSAN e VeSPA) per dargli qualche occupazione la visita dei convalescenti di ottalmia. Nessun ammalato di prima invasione ebbe a curare, o se pure qualche ricaduto, solo temporariamente ed abusivamente, giacché era in dovere di rimandarlo tosto nella sala ordinaria della cura. L'ottalmia fu sempre negli ospitali militari del Regno d'Italia di esclusiva pertinenza chirurgica. L'ispettore di sanità o li trattava alla medicazione direttamente, o i suoi chirirughi subalterni, tra i quali primeggiava per rango e per cognizioni il Prof. Bongrovanni ${ }^{19}$ morto pochi anni addietro sulla cattedra d'ostetricia nella R. Università di Pavia. Una confutazione aveva preparato il Dr. Rima; ma da che si vidde prevenuto dal Dr. OмоDEI, dimise il pensiero di pubblicarla ${ }^{20}$. Il libercolo pubblicato col nome del

19 Paolo Bongiovanni (1777-1827) percorse la carriera nell'esercito napoleonico e fu per cinque anni chirurgo in capo e professore di clinica chirurgica nell'ospedale militare d'Ancona. Nel 1819 ottenne per concorso la cattedra d'ostetricia nell'Università di Pavia.

${ }^{20}$ Pubblicò invece integralmente (Biblioteca Italiana 7, 97-101, 1817) due lettere dello Scarpa, la prima indirizzata al Ministro della Guerra il 13 novembre 1812 e mandata in copia al Rima quale ispettore di sanità in Ancona, la seconda inviata al RrMa stesso il 14 dicembre 1812. L'OмоDEI, cui il RIMA aveva in un primo tempo chiesto ospitalità negli «Annali», rimase dispiaciuto della cosa e si decise infine a ripubblicare le lettere, aggiungendo amare parole sia verso il Rima che verso il direttore della «Biblioteca 
Dr. VASANI era sopra gl'informi suoi materiali, stato scritto nelle prigioni di Mantova da Giovanni Rasori. Ciò che fu curioso si è che il Dr. Rima è stato il canale intermediario per cui passò il compenso pecuniario alla figlia dello scrittore. Se non emergeva la verità, risultavano in quel libercolo i meriti del recondito scrittore.»

Abbiamo già ricordato che nel 1815/16 il RASORI si trovava incarcerato nelle prigioni di Mantova, mentre la sua diletta SABINA versava in condizioni d'estrema povertà. Queste circostanze sembrano avvalorare l'affermazione del Rima che la redazione della Storia del VASANI sia stata curata dallo stesso RAsori. S'aggiunga poi, come nota lealmente il Rima e come anche il lettore avrà potuto direttamente costatare in base ai brani qui riportati, che la stesura dell'opera, così ricca di logica, non è certo opera di persona spregevole. Risulta poi dall'autobiografia che il RIMA conviene con l'OMODEI nel rivendicare a sé stesso la priorità degli esperimenti di trasmissione all'animale $^{21}$.

Ma lasciamo da parte le polemiche e cerchiamo, sia pure schematicamente, d'inquadrare l'episodio dell'oftalmia d'Ancona nella storia del contagio vivo.

Il concetto d'esalazione pestilenziale, d'un «quid vitiosum» esistente

Italiana» (Ann. Univ. di Med. 3, 385-98, 1817). Le due lettere sono anche riprodotte in Opere del Cav. Antonio Scarpa, prima edizione completa...per cura del D. Pietro Vannoni, Firenze 1, 231/2 (1836); e in A. Scarpa, Epistolario, Pavia 1938, p. 399-402.

${ }^{21}$ In seguito il Rrma (Sul Coléra, cenni critici, Giornale per servire ai progressi della Patologia e della Materia medica 3, 27-46, Venezia 1835) non sembrò alieno dall'identificare il contagio coleroso con minutissimi insetti impercettibili ad occhio nudo e previde, sia pur vagamente, la funzione patogena delle tossine: «Ma anche morti (gli insetti) il loro principio deleterio come avviene della cantaride potrebbe agire sui centri nervosi, e cagionare i sintomi mortali, e la morte stessa» (p. 44/5). Inoltre un allievo del Rima, Michelangelo Asson (1802-1877) - dopo aver accuratamente studiato in collaborazione con Cortese, Fario e Pancrazio il quadro clinico e anatomico della malattia (Osservazioni intorno alla prima invasione del Cholera-morbus in Venezia, Annali Univ. di Med. 78, 417-52, 79, 78-113, 1836), pubblicò un lavoro (Intorno al principio cholerico, e al modo con cui questo opera sull'organismo vivente, Commentarii di Medicina [opera periodica di G.F.Spongia, Padova] 1, 589-602, 731-86, 1836) in cui, ricordati gli analoghi esperimenti d'inoculazione nell'animale compiuti con vario esito da MAGENDIE, RAYER, Namias, Novati, Calderini, Semmola, riferì i propri tentativi infruttuosi per riprodurre la malattia nel coniglio, nel cane, nella cavia e nel pollo innestando sangue ed altri umori di coleroso; nel pollo tentò anche il trapianto in serie. Giunse alla conclusione che «Il principio choleroso, contagioso per gli uomini, non lo è similmente per varii animali» e che la teoria del contagio vivo, pur essendo logica, mancava ancora d'una dimostrazione obiettiva. 
nell'aria in tempo d'epidemia (più tardi chiamato «miasma») ${ }^{22}$ fu ammesso dai grandi medici dell'antichità (IPPOCRATE, ecc.) e, nel $\mathrm{I}^{\circ}$ secolo av. $\mathrm{Cr}$. assunse la fisionomia dei «semina» con Lucrezıo e di «animalia quaedam minuta» con VARRONE. Nel Rinascimento queste vaghe intuizioni trovarono logica formulazione nella dottrina dei «seminaria» di Girolamo FraCASTORO, dottrina in questo stesso volume giubilare esposta magistralmente da Arturo Castiglioni.

L'invenzione del microscopio, avvenuta agli albori del Seicento, fornì agli studiosi l'arma per andare alla ricerca degli «animalcula» produttori dei contagi: osservazioni in tal senso furono tentate da A. Kircher (1658) e da A. VAllisneri sen. (1714) ${ }^{23}$. Frattanto la scienza compiva tre conquiste d'importanza fondamentale per la dottrina del contagio vivo:

1) la scoperta dei microrganismi (A.v.LeEUWENHOEK, 1676 e 1683);

2) l'abbattimento della teoria della generazione spontanea, grazie agli esperimenti di F. Redi (1668) sugli insetti e di L. Spallanzani (1767) sugli Infusori;

3) la scoperta della natura parassitaria (Acaro) d'una malattia tipicamente trasmissibile quale è la scabbia (C. Bonomo e D. Cestoni, 1687).

Queste costatazioni sperimentali collegate ai risultati dell'osservazione epidemiologica rendevano sempre più logica alla mente di parecchi studiosi la dottrina del contagio vivo. Agli inizi dell'Ottocento mancava soltanto la dimostrazione sperimentale che la rendesse vera anche ai loro occhi. Quanto fosse sentito il contrasto fra la logica costruzione di questo edificio dottrinale e la mancanza d'un fondamento sperimentale, risulta con particolare evidenza dall'opera di Enrico Acerbi (1785-1827) ${ }^{24}$.

Proprio in questo periodo viene alla luce l'opuscolo del VASANI, il quale s'illuse d'aver portato la dimostrazione sperimentale alla dottrina del contagio vivo professata dal suo maestro RASORI. Gli elementi interessanti di

${ }^{22} \mathrm{C}$. Frugoni, Le antiche teorie miasmatiche alla luce delle moderne conoscenze sugli aerosols, Humana Studia 1, 59-68 (1949).

${ }^{23}$ Sui seguaci settecenteschi della dottrina del contagio vivo, come C.F. Cogrossi (1714) e B. Corte (1720), e in generale sulla storia dell'argomento segnalo gli importanti lavori di U. FAucci pubblicati sulla Riv. Storia Scienze Med. Natur.: Contributo alla storia della scabbia $(22,153-70,198-215,257-371,441-75,1931)$. Contributo alla storia della dottrina parassitaria delle infezioni: sua connessione colla scoperta dell'origine acarica della scabbia (25, 27-70, 207-65, 1934, 26, 136-93, 243-64, 309-46, 1935). In memoria di Agostino Bassi, nel $1^{\circ}$ Centenario del «... Mal del Segno» (27, 1-26, 59-102, 153-206, 286-326, 371-423, 1936, 28, 24-37, 283-305, 1937, 30, 85-98, 209-28, 1939, 32, 1-32 (1941).

${ }^{24}$ E. ACERBI, Dottrina teorico-pratica del morbo petecchiale, con nuove ricerche intorno l'ori- 
quel lavoro possono esser suddivisi in tre gruppi, per altro strettamente collegati fra loro:

1) studio epidemiologico, ricco di acute osservazioni sulla trasmissione e la latenza del contagio;

2) esperimenti di trasmissione del contagio all'animale e precisamente: trasmissione diretta dall'uomo all'animale; trasmissione da animale ad l'animale (esperimenti con l'acqua di lavaggio); trasmissione indiretta al animale mediante veicoli infetti (esperimenti col pus disseccato e ridotto in polvere);

3) identificazione, sia pure errata, dell'agente patogeno, tanto nel pus umano che in quello proveniente dall'animale artificialmente infetto.

Per chiudere l'anello mancava soltanto l'isolamento in coltura pura e la trasmissione della malattia col germe coltivato.

Cosa vide realmente il VASANI? In una postilla al termine dell'opera, egli afferma «il Microscopio, di cui mi sono servito per esaminare il pus ottalmico, ingrandiva 1250 volte». $\mathrm{E}$ verosimile che si tratti d'ingrandimento di superficie anzichè lineare; pertanto il VASANI avrebbe compiuto le sue osservazioni a un ingrandimento di 35 diametri. Con ciò il normale diametro $(10-12 \mu)$ d'un granulocita neutrofilo veniva portato a $350-420 \mu$, ossia a una grandezza aggirantesi sulla «dimensione d'un mezzo grano di miglio» di piccola taglia. Malgrado la forma conico-schiacciata, i corpuscoli osservati dal VASANI e da lui interpretati quali agenti patogeni dell'oftalmia, erano evidentemente dei piociti.

Sono gli stessi «globuli» osservati nel 1805 da Michele Francesco Buniva (1761-1834) ${ }^{25}$ nel pus vaioloso e vaccinico. Anche il Buniva aveva intrapreso quelle osservazioni microscopiche per identificare gli «animalculas del contagio, ma, dotato di maggior senso critico del VASANI, aveva concluso in senso negativo la sua ricerca.

Nel 1809 gli stessi piociti o "granelli della marcia» furono descritti e raffigurati da Franz von Paula Gruithuisen (1774-1852) ${ }^{26}$ nel corso di

gine, l'indole, le cagioni predisponenti ed effettrici, la cura e la preservazione del morbo medesimo in particolare, e degli altri contagi in generale, Milano 1822.

${ }^{25}$ Résultats détachés de quelques recherches expérimentales sur les phénomènes de l'infection et de la désinfection tant spontanée qu'artificielle; et notamment sur ... l'inexistence des animalcules microscopiques dans la matière vaccinale ..., Mémoires de l'Académie Impériale des Sciences, Littérature et Beaux-Arts de Turin, pour les années 1805-1808; Sciences physiques et mathématiques, Turin 1809, p. 127-40.

${ }^{26}$ Naturhistorische Untersuchungen über den Unterschied zwischen Eiter und Schleim durch das Mikroskop, München 1809. Il lavoro fu anche tradotto in italiano da C. Ceresa: 
ricerche microscopiche tendenti a stabilire un criterio differenziale tra pus e muco in base allo studio dei microrganismi che compaiono nelle infusioni delle due sostanze.

Nello stesso anno, lo «Jenner italiano», Luigi SAcco (1769-1836), che già s'era dimostrato propenso alla dottrina del contagio vivo ${ }^{27}$, pubblicò l'opera che doveva rimaner classica nella storia della vaccinazione in Italia ${ }^{28}$ e che racchiude tra l'altro i risultati delle ricerche microscopiche da lui compiute sul pus vaccinico. In questa sostanza il SACCo osservò la presenza di «globetti», di forma un po' allungata nel vaccino vero e più rotonda in quello spurio, dotati di «una specie di movimento vermicolare» e costatò che il loro numero era proporzionale all'attività del vaccino: nella «linfa» spontaneamente inattiva o quasi essi erano molto rari $e$ in quella inattivata col calore o con gli acidi erano completamente scomparsi. Anche il VASANI s'era proposto di studiare il parallelismo tra il potere contagiante del pus e la quantità degli «animaluzzi» in esso contenuti.

Tralascio gli esperimenti col calore, che tanta parte avevano avuto nella celebre polemica sulla generazione spontanea degli Infusori, per ricordare le osservazioni del SACco sul pus disseccato: «. . la goccia di materia si era disseccata; vi aggiunsi quindi una goccia di acqua fredda col mezzo di uno stecchetto, e scorrendo coll'occhio i diversi punti che si andavano sciogliendo dall'acqua, vidi ricomparire di mano in mano gli stessi corpicini, della stessa figura, e col medesimo movimento, che spariva no al mancar dell'umido ... Raccolsi due gocce di umor vaccino limpido su due lamine divetro, e differii ad altro giorno la osservazione per esaminare ciò che rappresentava lo stesso umore disseccato. Dopo tre giorni potei scorgere ad occhio nudo, che la materia seccatta era trasparente, si era screpolata irregolarmente, e mi offeriva tante squamette ineguali, che escludevano qualunque siasi idea di cristallizzazione. Esaminata tale materia colla lente ordinaria osservai alcuni profondi solchi impressi su di un corpo semitrasparente: questi solchi erano le screpolature della materia secca, le quali anche ad occhio nudo si distinguevano. Con il solito stecchetto vi portai una goccia di acqua fredda, e vidi di mano in mano che si faceva la soluzione, ricomparire i globetti, ed acquistare una

Ricerche fisiche sulla differenza, che passa fra il muco e la marcia, per mezzo del microscopio, Anm. Med. Straniera 3, 147-74 (1815).

${ }^{27}$ Osservazioni pratiche sull'uso del vajuolo vaccino, come preservativo del vajuolo umano, Milano, anno IX rep. (1800), p. 58/9.

${ }^{28}$ Trattato di vaccinazione con osservazioni sul giavardo e vajuolo pecorino, Milano 1809, p. 179-83. 
specie di movimento. Sottoposi alla osservazione un poco di materia secca, che senza riguardo io aveva lasciata per alcuni mesi esposta all'aria. Ho replicato $\mathrm{e}$ in diversi modi variato la esperienza, ma non mi riuscì mai di vedere in questa materia tutto quell'ammasso di globetti, e talvolta nessuno o ben pochi ne apparivano.»

A nessuno sarà sfuggita la spiccata analogia tra queste osservazioni del SAGco e quelle del VASANI, il quale sottolinea in proposito il paragone con la «resurrezione» del Rotifero. Questo fenomeno, pochi decenni prima chiaramente lumeggiato anche in Italia per opera di L. SpALlANZANI ${ }^{29}$, era stato invocato dal RASORI, assieme alla deposizione d'uova, per spiegare la latenza dei contagi.

Le osservazioni microscopiche del VASANI non peccano quindi d'eccessiva originalità, a parte il fatto che, per usare le parole del Pollini ${ }^{17}$, egli rimase «allucinato, siccome occorre spesse fiate, massime a chi non è accostumato a simil fatta d'indagini».

Oggi i protagonisti della nostra storia vagolano nei campi eterni: il VASANI che per combattere «l'error de' ciechi» volle troppo vedere, il RASORI che forse, nella durezza del carcere, diede veste letteraria agli appunti dell'allievo, il Rima così blando nel rivendicare la priorità dei propri esperimenti sugli animali, l'OмоDeI che s'erse a paladino dell'onore vilipeso del corpo sanitario militare. Il silenzio della morte ha posto fine alle aspre polemiche e il segreto della tomba ci vieta d'attribuire a ciascuno il suo. A noi interessa soprattutto l'episodio in sè stesso: ricco di geniali intuizioni, d'acute osservazioni e di banali errori, esso è un piccolo anello della lunga catena verso le grandi scoperte della seconda metà dell'Ottocento, che schiusero una nuova èra alla medicina e all'umanità : l'èra batteriologica.

${ }^{29}$ Osservazioni e sperienze intorno ad alcuni prodigiosi animali che è in balìa dell'osservatore il farli tornare da morte a vita, Opuscoli di fisica animale e vegetabile..., Modena 1776. 